\title{
Mise en invisibilité des individus et reconnaissance des activités
}

Un cas d'implantation du dossier patient numérique

\section{Benoit Cordelier}

\section{OpenEdition \\ Journals}

Édition électronique

URL : http://journals.openedition.org/communicationorganisation/4289

DOI : 10.4000/communicationorganisation.4289

ISSN : 1775-3546

Éditeur

Presses universitaires de Bordeaux

Édition imprimée

Date de publication : 1 décembre 2013

Pagination : 29-40

ISBN : 978-2-86781-878-3

ISSN : $1168-5549$

\section{Référence électronique}

Benoit Cordelier, "Mise en invisibilité des individus et reconnaissance des activités », Communication et organisation [En ligne], 44 | 2013, mis en ligne le 01 décembre 2016, consulté le 01 mai 2019. URL http://journals.openedition.org/communicationorganisation/4289; DOI : 10.4000/ communicationorganisation.4289 


\title{
Mise en invisibilité des individus et reconnaissance des activités
}

\author{
Un cas d'implantation du dossier patient numérique
}

\author{
Benoit Cordelier ${ }^{1}$
}

Pour Axel Honneth (2004, 2005, 2013), la reconnaissance s'appuie à la fois sur l'identification cognitive de l'individu et l'expression publique de celle-ci. Les modalités de cette confirmation sont importantes pour reconnaître la valeur sociale des individus. Faudrait-il encore définir les critères de cette valorisation. Dans le contexte organisationnel, ils sont de toute évidence rattachés à la contribution que la personne apporte aux activités. Mais pour que cette contribution soit visible, il faut qu'elle résolve une tension perçue. Les enjeux de résolution peuvent varier selon les contextes et nous allons montrer que, dans le cas des processus projets en particulier, elle peut signifier un affaiblissement de la reconnaissance.

Nous abordons cette problématique à travers l'étude d'un terrain où une organisation de santé passe d'un système de dossiers patients papier vers des dossiers numérisés. Nous nous concentrons dans cette optique sur le double travail de mise en visibilité et invisibilité des processus organisationnels (et donc du travail) qui touchent en particulier le personnel du service des archives de ce centre de santé.

Les interactions pragmatiques (Gramaccia, 2001) en contexte de projet de changement organisationnel transforment les modalités de reconnaissance (Honneth, 2004) par la mise sous tension des acteurs à travers des dispositifs normatifs (Cordelier, 2008, 2012a) qui cherchent à inscrire les acteurs organisationnels dans de nouvelles trajectoires de travail (Strauss, 1992). Ces trajectoires organisationnelles (Cordelier, 2012b) imposent en somme des

\footnotetext{
1 Benoit Cordelier est professeur au Département de communication sociale et publique de l'Université du Québec à Montréal (UQAM), chercheur à la Chaire de relations publiques et communication marketing et au Centre de recherche sur la communication et la santé (ComSanté) ainsi que chercheur associé au MICA (EA 4426, axe Communication, organisations et société, Université Bordeaux Montaigne), directeur de la Revue internationale de communication sociale et publique (RiCSP) de l'Université du Québec à Montréal (UQAM), Canada. Ses recherches portent sur les processus organisationnels, le lien social dans les communautés de consommation et la relation aux objets et marques ou encore le branding des universités.
} 
critères de reconnaissance temporaires liés au projet et limitent les possibilités de discuter les conséquences de la transformation des processus de travail. En effet, si les activités de transformation s'appuient sur un régime d'exception qu'est le projet, celui-ci reste subordonné au bon déroulement des activités usuelles de l'organisation, autrement dit à l'opération. Ainsi, les archivistes de l'organisation étudiée participent à la disparition de leur propre activité à travers des procédures temporaires, ou régulations de conversion (Cordelier \& Gramaccia, 2006), dont les accidents mettent en évidence "l'invisible matérialité de leur travail quotidien" (Le Bis \& Vacher, 2006). Ces processus organisationnels permettent de répondre à des contradictions (Yrjö Engeström, 1987, 2001) et contribuent au développement de l'organisation. Les services de direction mettent en place des dispositifs normatifs et communicationnels qui permettent l'émergence de la reconnaissance (Andonova \& Vacher, 2009) mais la reconfiguration des processus et des activités professionnelles qui s'y inscrivent amène également des changements dans les modalités de reconnaissance et un déplacement du contexte dans lequel elle se réalise.

Dans un premier temps, en nous appuyant sur la théorie de l'activité, nous nous attardons sur la notion de contradiction qui nous apparaît essentielle pour identifier la valeur attribuée à une activité, et donc aux individus qui la soutiennent. Dans un deuxième temps, nous exposons notre terrain avec le souci de présenter le contexte dans lequel s'insèrent les employés du service archives sur lesquels porte notre analyse. Et nous terminons, par une mise en relation du mécanisme de résolution des contradictions produisant une relation paradoxale entre mise en visibilité et reconnaissance de leur travail.

\section{Du travail à l'activité et à sa mise en invisibilité}

La théorie de l'activité a été d'abord élaborée par Vygotski (1978 [1934]) dans le premier tiers du $\mathrm{XX}^{\mathrm{e}}$ siècle avant qu'elle ne soit reprise par Alexei Leontiev. Engeström (2001) identifie par la suite trois générations de théories de l'activité.

La première approche de Vygotski développe l'idée de médiation en s'appuyant sur son modèle triangulaire qui établit une connexion directe entre stimulus et réponse enchâssés dans un contexte culturel qui oriente la capacité d'agir des individus en les amenant à produire et à utiliser des artefacts culturels. Vygotski tente ainsi de réduire la séparation entre l'individu et la structure sociale. Suite à cela, il propose d'étudier les individus à travers leurs médiations culturelles (autrement dit, l'influence de la société) et la société à travers l'agentivité des individus qui se déploie grâce à leur production et l'usage d'artefacts. Toutefois, selon Engeström (2001), l'individu reste l'unité d'analyse de ce modèle.

La deuxième génération se poursuit autour de Leontiev et différencie l'action individuelle de l'action collective. Selon Engeström (2000, 2001), il parvient ainsi à dépasser la vision centrée sur l'individu (Kaptelinin, 2005) 
car si l'approche psychologique originale peut laisser croire que l'individu est toujours central, Leontiev (1978 [1975]) précise que l'activité individuelle doit toujours être prise en compte dans le cadre de relations sociales ${ }^{2}$.

La troisième génération de la théorie de l'activité s'est attachée à construire les outils conceptuels pour comprendre le dialogue obligatoire entre les différents systèmes d'activité en interaction. Elle s'y est consacrée en introduisant les idées de la dialogique de Bakhtine pour approfondir l'approche de Vygotski (Wertsch, 1993), en les reliant aux conceptions de l'activité selon Leontiev (R. Engeström, 1995) et en introduisant le concept du réseau d'activités (Russell, 1997).

La théorie de l'activité est alors prête pour envisager l'interaction, les échanges entre plusieurs systèmes d'activité, dont les objets ou visées ${ }^{3}$ font l'objet d'une négociation qui se produit par des tensions entre les systèmes. Elle s’intéresse donc aux contradictions systémiques (Yrjö Engeström, 2001).

Pour Engeström (1999), la théorie de l'activité propose les bases conceptuelles et méthodologiques de l'étude de la visibilité du travail. L'activité collective se conçoit à travers un motif commun lorsque « un besoin collectif rencontre une visée " (Yrjö Engeström, 1999, 65). Cette dernière porte alors le motif et doit être comprise comme un projet. Elle détermine l'objectif et les actions à entreprendre pour l'atteindre. Une même action peut également permettre la poursuite de plusieurs objectifs et correspondre à plusieurs activités. De la même manière, le motif et la visée d'une activité collective peuvent être atteints de diverses façons à travers des actions et des objectifs (intermédiaires) alternatifs. Ce point amène dès lors la possibilité de voir émerger des contradictions dans la poursuite d'une visée. Par exemple, le Dossier patient numérisé est une activité au service de l'objectif général de soin des patients mais l'introduction d'une nouvelle technologie et la réorganisation des processus qui s'en suit se heurtent aux règles de fonctionnement et activités en place. En fait, pour Engeström la visée de toute activité porte en elle-même une contradiction interne qu'il illustre par ce qu'il appelle, en rappelant les racines marxistes de la théorie de l'activité, la contradiction primaire du capitalisme entre la valeur d'usage et d'échange d'un objet. Cette contradiction est censée évoluer, mais pas forcément se résoudre, dans une zone (floue) de développement proximal qui crée des contradictions

2 The basic, constituent feature of activity is that it has an object. In fact, the very concept of activity (doing, Tätigkeit) implies the concept of the object of activity. The expression "objectless activity" has no meaning at all. Activity may appear to be objectless, but the scientific investigation of activity necessarily demands the discovery of its object. Moreover, the object of activity appears in two forms: first, in its independent existence, commanding the activity of the subject, and second, as the mental image of the object, as the product of the subject's "detection" of its properties, which is effected by the activity of the subject and cannot be effected otherwise. (Leontiev, 2009 [1975])

3 Le modèle d'Engeström utilise l'expression objet pour parler de l'objectif, de la visée d'un système d'activité. Le terme peut prêter à confusion. Cette difficulté est héritée de l'influence de la philosophie allemande sur la psychologie russe et des traductions des travaux de Leontiev vers l'anglais (Kaptelinin, 2005). Pour la contourner, nous lui préférerons ici le terme de visée. 
secondaires entre différents systèmes d'activité. Dans la théorie de l'activité, les visées viennent s'appuyer sur les éléments de la médiation sociale de l'activité que sont les règles, la communauté et la division du travail. Pour Engeström, les contradictions ne se manifestent pas directement mais par des perturbations, des ruptures ou de petites innovations dans les actions quotidiennes que le chercheur doit rendre visibles pour pouvoir les étudier. De la même manière, nous constatons que pour pouvoir agir, les individus d'une organisation doivent pouvoir identifier les oppositions ou antinomies qui les inscrivent dans des systèmes d'activité à la légitimité variable. Cela pose le problème de la visibilité des systèmes d'activité non seulement pour les chercheurs mais également pour les acteurs de l'organisation.

Pour véritablement intéressantes que nous apparaissent la théorie de l'activité et la richesse que nous trouvons dans la notion de contradiction, nous remarquons toutefois que le modèle proposé par Engeström, et largement repris par d'autres chercheurs (p. ex. : Blackler, Crump, \& McDonald, 1999 ; Bonneau, 2011; Foot \& Groleau, 2011; Groleau, 2005, 2008), ne souligne pas assez la cohabitation d'activités concurrentes n'ayant pas le même degré de légitimité en fonction de leur intégration dans une trajectoire organisationnelle plus large (Cordelier, 2005, 2012a). Si nous pouvons évidemment penser à la distinction entre des activités formelles et informelles, nous insistons plutôt ici sur les antinomies qui peuvent émerger entre les figures du projet et de l'opération, ce que nous retrouvons dans les étapes du projet de numérisation du dossier patient que nous avons étudié. Dans notre cas, les archivistes participent à la mise en place d'un système informatisé fortement soutenu par les institutions politiques et administratives qui en intégrant les processus d'archivage fait disparaître leur emploi. L'activité d'archivage est nécessaire (légitime) mais l'unité organisationnelle qui la représente n'apparaît plus aussi importante qu'elle a pu l'être.

\section{La numérisation du dossier patient}

Dans la plupart des pays de l'OCDE, l'amélioration du système de santé passe par l'amélioration des processus de soin en tirant profit d'un développement des systèmes d'information s'appuyant sur l'intégration de technologies de l'information. Le gouvernement du Québec incite à des projets d'informatisation du réseau régional et de dossier de santé électronique. Les thématiques de rationalisation, de standardisation, de rentabilité des activités de soins, de continuité des soins et d'accessibilité aux informations parcourent le projet Dossier santé du Québec (DSQ) qui s'inscrit dans ces perspectives.

Le dossier de santé électronique (dossier clinique, dossier patient, etc.) touche à la question de la structuration des organisations et, par ricochet, à la réorganisation des processus, des pratiques professionnelles et des professions, elles-mêmes impliquées dans les systèmes de soin. Nous avons observé ces transformations à travers un cas d'implantation du Dossier patient numérisé 
(DPN) dans un Centre de santé et de services sociaux (CSSS) montréalais. C'est une étape intermédiaire dans un projet plus large identifié par l'acronyme OACIS. Nous avons plus particulièrement étudié la mise en place du module de visualisation des dossiers et la mise en place du processus de numérisation des données.

Sans rentrer dans les détails de la présentation des parties prenantes au projet (SSII, institutions publiques, administrations...) et du déroulement $\mathrm{du}$ dossier, nous pouvons apporter quelques précisions méthodologiques. Le projet touche les activités de 800 employés du CSSS. Il s'appuie sur 22 groupes de travail dans lesquels participent directement 80 personnes. Les groupes de travail s'occupent de l'adaptation des formulaires et des processus pour préparer la future application. Avant d'aller vers un dossier clinique informatisé (DCI), l'organisation passe par un dossier patient numérisé (DPN). L'implantation est déclenchée avec une approche «Big Bang »".

Notre intervention se déroule après le déploiement, avec pour objectif de recueillir les discours des usagers d'OACIS. En parallèle d'une étude documentaire des outils de communication d'accompagnement du changement préparés par la Direction de l'organisation, nous avons opté pour une intervention à base d'entretiens semi-dirigés afin de recueillir des informations diverses comme des faits, des opinions, des analyses personnelles, des réactions, des propositions. La sélection des personnes à interroger a fait l'objet de plusieurs ajustements pour que nous puissions nous adapter aux réalités du terrain. Cela a abouti à la production de quatre groupes de synthèses d'entretiens pour un total de 48 personnes interrogées :

- Comité de direction du projet, usagers administratifs (8 personnes)

- Comité de direction du projet, usagers cliniques (5 personnes)

- Comités processus (20 personnes)

- Usagers hors-projet (15 personnes)

Le questionnaire visait principalement à recueillir le point de vue des employées du CSSS afin d'obtenir leurs discours sur leur réception du projet DCI et sur les actions de conduite du changement (communication, formation). L'objectif secondaire du questionnaire était de cerner les pistes d'amélioration qu'ils envisageraient. Les entretiens ont été conduits plus de neuf mois après le déploiement du module de visualisation faisant partie de la première livraison.

Si dans le modèle d'Engeström, l'activité est l'unité d'analyse, il faut préciser que notre accès au terrain s'est essentiellement fait au travers d'une étude documentaire et d'entrevues. Ces dernières rendent compte des perceptions qu'ont les acteurs du projet et viennent témoigner de leurs motivations et

4 Ce type d'approche favorise un déploiement rapide afin de mobiliser les ressources pendant une courte période car, généralement, elles ne sont pas disponibles sur un plus grand délai. L'effort est intense afin de passer plus rapidement à travers les difficultés et le traumatisme du changement. Ce dernier est toutefois généralement plus intense avec cette méthode. 
des actions qui en découlent. Les productions discursives nous permettent également de reconstituer une histoire collective du processus étudié et d'éclairer le contexte organisationnel du projet en explicitant les logiques à l'œuvre tout en les inscrivant dans les différents systèmes d'activité des acteurs. S'il eut été souhaitable d'aborder le terrain à travers des méthodes d'observation plus directe, cette approche nous a malgré tout permis de respecter en grande partie les indications méthodologiques suggérées pour la théorie de l'activité, notamment la dimension historique (Yrjö Engeström, 2001 ; Miettinen \& Hasu, 2002), et de tester les hypothèses qui surgissaient en adaptant nos questions à mesure que se déroulaient les entretiens conformément à une approche de théorisation ancrée (Strauss \& Corbin, 1994).

\section{Mises en (in)visibilité : antinomie de rôles au service des archives}

En ce qui concerne notre travail de terrain sur le projet OACIS, il a été réalisé après le déploiement du visualiseur (première livraison du projet) qui a supposé la numérisation préalable des archives et des dossiers suivis pendant la période de transition. L'enjeu de cette étape était de parvenir à numériser les archives et permettre la transition des dossiers en cours en minimisant le temps de cohabitation des dossiers papier avec les dossiers numérisés. Deux éléments nous sont apparus essentiels dans les questions de mise en (in) visibilité du travail. Le premier relève d'un processus informel qui pourrait être qualifié de système d'action concret (Crozier \& Friedberg, 1977) : pour pallier ce que les employés perçoivent comme des dysfonctionnements, notamment dans le processus d'archivage, des dossiers parallèles transitoires apparaissent. Le deuxième est typique des processus de rationalisation de l'organisation qui s'appuie sur le service des archives et aboutit à la suppression de leurs postes. Pour des questions de place, nous nous concentrerons sur ce dernier. Il nous apparaît en effet d'autant plus intéressant, dans le cadre de ce dossier, que les processus étudiés articulent de manière éclatante une relation dynamique paradoxale entre reconnaissance et mise en visibilité du travail des archivistes.

Le début de la procédure de numérisation des dossiers patient connaît quelques accidents, en particulier en ce qui concerne les dossiers historiques. Au lancement du projet sur le terrain, des dossiers archivés sont indisponibles pendant de longues semaines. La numérisation des dossiers en cours pose quant à elle quelques défis autour des délais de traitement. Si ces derniers sont théoriquement rapides, il peut arriver que des patients reviennent avant que leur dossier n'ait été enregistré et rendu disponible dans le système. De plus, lors de la numérisation certains dossiers ont pu être altérés (inversion de pages, par exemple). Si ces accidents ont pu être corrigés, ils mettent en évidence l'importance du service des archives à la fois dans le fonctionnement quotidien de l'organisation comme dans le déroulement temporellement plus limité du projet. Avant la numérisation, il faisait le lien entre les différents métiers et antennes du CSSS lorsqu'il s'agissait de rendre l'information 
disponible. Les tâches des employés des archives étaient relativement variées. Ils étaient au contact de nombreuses personnes pour assurer la transmission des dossiers. Le service a pris une importance accrue dans cette phase du projet OACIS. Les dossiers patients passent par lui pour la numérisation. Il en garantit le bon traitement et est même amené à proposer de nouvelles idées pour en améliorer le fonctionnement. Toutefois, la nature du travail qu'il effectue devient plus monotone et répétitive en raison, dans un premier temps, des volumes d'information et, dans un deuxième temps, de la diminution de l'importance de la fonction d'interface de ses membres puisqu'elle est peu à peu assurée par le nouveau système informatisé. Cela aboutit à une rotation du personnel plus grande et à une réduction du service par des coupures de postes. Pour conserver une place dans l'organisation, des employés se voient proposer des réaffectations qui sont diversement appréciées.

La distinction des fonctions tenues par les archivistes à travers leurs activités d'opération puis de projet nous permet de constater que la valorisation de leur contribution vient de l'intégration de leurs travaux dans l'ensemble des processus organisationnels soumis au système d'activité principal, celui de soin. La valeur d'usage de la fonction d'archivage est importante pour poursuivre la visée et l'activité de soin. Sa valeur d'échange en revanche va se réduire à mesure que cette activité peut être automatisée, intégrée et devenir idéalement transparente. Si la discrétion, pour reprendre un terme de Le Bis et Vacher (2006), peut être un signe de la qualité des prestations, c'est dans l'accident qui rompt avec cet idéal que l'importance du travail des archivistes est rappelée et peut alors être reconnue. Si, comme nous l'explique Honneth (2004, 2005, 2013), la reconnaissance s'appuie sur l'identification cognitive de l'individu et sa reconnaissance publique, nous aboutissons à une situation paradoxale où, pour être valorisé et surtout reconnu, le processus dans lequel s'inscrit le travail de l'individu doit faire l'objet d'un loupé, d'un dysfonctionnement. Dans notre cas, nous en identifions de deux ordres. Le premier est celui que nous pourrions appeler l'accident ordinaire. Il fait partie de l'opération et peut se traduire par un délai inhabituel dans la mise à disposition de l'information suite, par exemple, à une erreur occasionnelle d'archivage, au retard ou à l'absence d'une personne impliquée dans le processus. Le deuxième s'inscrit dans une transformation des processus faisant l'objet d'une innovation organisationnelle, souvent dans une logique de projet qui œuvre à la rationalisation d'une organisation. Dans ce cadre, la gestion des contradictions de l'organisation entraîne inévitablement la production de perturbations (processus temporaires de changement, attribution temporaire de tâches reliées au projet, réaffectation d'employés, réorganisation des services...) dont la résolution globale est programmée sans que, concrètement, ne puissent être écartés tous les risques d'accident.

La légitimité des archivistes dans le système de soin se construit sur leur aptitude à classer et à faciliter la circulation des dossiers patients. Autrement 
dit, ils ont une place, un rôle utile dans l'organisation. Leur fonction y a une valeur d'usage. Une perturbation (Yrjö Engeström, 1996 ; Norros, 1996) vient pourtant transformer radicalement leur rôle : l'implantation du DPN. Ils deviennent alors une pièce essentielle dans les opérations de transition. Ils gagnent en visibilité par leur participation essentielle au bon déroulement du nouveau processus projet et y acquièrent une nouvelle valeur d'échange. Au fur et à mesure de l'avancement dans la trajectoire organisationnelle qui mène à la réorganisation du dossier patient, l'ordre social se reconstruit (Strauss, 1992) en fonction de contradictions (Yrjö Engeström, 1999, 2001) que les activités cherchent à dépasser. La capacité des archivistes à résoudre ces contradictions contribue à maintenir temporairement une forme de légitimité liée à l'utilité temporaire, en sursis, qu'ils représentent. La contradiction subie et résolue par les archivistes leur permet de voir leurs valeurs d'usage et d'échange s'accroître temporairement. Elles diminuent pourtant à mesure que le projet progresse inexorablement vers son dénouement. Leur valeur est contextuelle et temporellement limitée, cadrée par le projet. Il devient dès lors nécessaire d'envisager de l'analyser dans une approche processuelle car le contexte évolue et donc la valeur de l'individu par rapport à l'activité, au processus évolue aussi en conséquence. C'est particulièrement frappant dans les figures du projet où les activités qu'il fomente sont appelées à disparaître. Le travail $\mathrm{y}$ est particulièrement visible tant qu'il aide à résoudre les contradictions de l'organisation. Cette tâche accomplie, il se transforme, disparaît ou retourne éventuellement vers une routine sans perturbations. La part de l'invisible s'accroît. La perception de la valeur diminue. Les modalités de reconnaissance s'en trouvent affectées et ne peuvent alors, de façon générale, qu'aller, elles aussi, en s'amenuisant. Elles prennent ici la forme d'une offre de reclassement qui ne s'explique plus seulement par la différence entre valeur d'usage et valeur d'échange. Mais elles n'ont pas immédiatement disparu.Le modèle d'Engeström permet de signaler que des contradictions avec d'autres systèmes d'activités voisins (contradictions quaternaires; $c f$. Engeström, 1987) peuvent apparaittre lorsque, dans le système d'activité central, les tensions s'apaisent. Pourtant, il n'est pas possible de prendre pour acquis que ce transfert de tensions se fait avec à l'esprit avant tout l'intérêt économique de l'organisation. Il faudrait en effet pouvoir débattre de la mesure de la valeur d'usage et d'échange de l'individu dans le système d'activité de réaffectation dans une perspective de transferts de compétence. Ne faut-il pas alors convoquer une dimension morale pour justifier cet effort ?

\section{Conclusion}

La contradiction, telle qu'elle est présentée par Engeström, permet de rendre visibles des activités et les individus qui les développent. Cette mise en visibilité aurait pu faciliter un travail de reconnaissance. Mais, par la contribution même de ces derniers à sa résolution, elles aboutissent 
pourtant avant tout à la disparition d'activités humaines au profit de leur intégration dans des dispositifs technologiques. Dans le cas qui nous occupe, non seulement les conditions de travail des archivistes se dégradent mais une réaffectation, pas toujours souhaitée, est à envisager en raison de la suppression du nombre de leurs postes. Leur rôle est absorbé par le dispositif OACIS qui normalise et intègre leur activité. Leur valeur pour l'organisation s'amenuise, pour ne pas dire s'éteint, avec la fin du projet. Dès lors, l'expression positive de la reconnaissance de leur contribution ne garde plus forcément un lien direct avec leur activité précédente et passe par un reclassement. Elle n'est pas le résultat exclusif d'un différentiel entre valeur d'usage et valeur d'échange qui conduirait à une exclusion de l'organisation. Elle est même repoussée en dehors du processus du projet. La perspective d'influence marxiste qu'adopte Engeström n'apparaît donc pas suffisante pour expliquer le mécanisme de reconnaissance à l'œuvre.

Pour Honneth (2004, 146-147), " [dans] l'acte de la reconnaissance, un décentrement s'opère chez le sujet parce quill concède à un autre sujet une "valeur" qui est la source d'exigences légitimes qui contrarient son "amour-propre" ". Le travail de mise en visibilité et invisibilité de l'activité d'archivage permet de résoudre une contradiction dans les systèmes d'activités tout en faisant disparaître la valeur des archivistes pour les dits systèmes. Pourtant la légitimité qu'ils ont cultivée et renforcée en étant un élément contributeur à cette résolution crée une dissonance morale que nous n'avons pas explorée ici mais qu'il serait important d'étudier plus en détail.

La reconnaissance à la fin des activités de projet peut évidemment être gérée de manière contractuelle dans une logique économique. Mais lorsque les relations sont déséquilibrées, et a fortiori en faveur de l'organisation, elle relève alors d'une logique morale qui s'intéresserait à la valeur sociale des individus. Il y a bien une forme de décentrement qui opère puisque la reconnaissance ne s'inscrit plus dans le système d'activité central mais serait transposée dans un autre service représentant un système d'activité voisin. Cette dernière semble pourtant devoir être ramenée dans le cadre d'une conception utilitariste qui la limite dans le temps pour en aligner rapidement les fins secondaires à un intérêt économique.

\section{BIBLIOGRAPHIE}

ANDONOVA Y \& VACHER B., "Visibilité et reconnaissance de l'individu au travail » in Communication \& Organisation, (2), 2009, 136-147.

BLACKLER F., CRUMP N. \& MCDONALD S., " Managing Experts and Competing through Innovation: An Activity Theoretical Analysis » in Organization, 6 (1), 1999, p. 5-31. doi:10.1177/135050849961001 
BONNEAU C., "Co-configuration d'une plateforme à code source ouvert en organisation : analyser la transformation d'un outil et des pratiques de travail avec la théorie de l'activité » in Revue Internationale de Communication Sociale et Publique, (5), 2011, p. 23-36.

CORDELIER B., "L'urgence : un outil de médiation locale dans les projets de changement organisationnel " in V. Carayol (Ed.), Vivre l'urgence dans les organisations (pp. 97-106). Paris: L'Harmattan, 2005.

CORDELIER B., "Between symbolic and regulationists transactions. For a new praxeology around the technical object ». Présenté au colloque « What is an Organization? Materiality, agency and discourse », HEC Montréal, LOG. 2008.

CORDELIER B., Changement organisationnel et management par projet: mobilisation des systèmes d'information. Paris: l'Harmattan, 2012a.

CORDELIER B., " Trajectoire organisationnelle et normativité souple: de l'importance du contexte dans les possibilités de discours » in B. Cordelier \& G. Gramaccia (Eds.), Management par projet: les identités incertaines (p. 103-114). Québec: Presses de l'Université du Québec, 2012b.

CORDELIER B. \& GRAMACCIA G., « Antinomies, régulations et médiations dans les projets d'innovation organisationnelle » in O. Germain (Ed.), De nouvelles figures du projet en management (pp. 99-123). Paris: EMS, 2006.

CROZIER M. \& FRIEDBERG E., L'acteur et le système. Paris: Seuil, 1977.

ENGESTRÖM R., "Voice as communicative action» in Mind, Culture, and Activity, 2(3), 1995, 192-215. doi:10.1080/10749039509524699

ENGESTRÖM Y., « Learning by expanding ». An activity-theoretical approach to developmental research, 1987.

ENGESTRÖM Y., "The tensions of judging: Handling cases of driving under the influence of alcohol in Finland and California » in Y. Engeström \& D. Middleton (Eds.), Cognition and communication at work (pp. 199-232). Cambridge: Cambridge University Press, 1996.

ENGESTRÖM Y., "Expansive Visibilization of Work: An Activity-Theoretical Perspective » in Computer Supported Cooperative Work (CSCW), 8(1-2), 1999, 63-93. doi:10.1023/A:1008648532192

ENGESTRÖM Y., "From individual action to collective activity and back: developmental work research as an interventionist methodology » in Luff \& al (Eds.), Workplace studies - recovering work practice and information system design. Press Syndicate of University of Cambridge, 2000.

ENGESTRÖM Y., « Expansive Learning at Work: Toward an activity theoretical reconceptualization " in Journal of Education and Work, 14(1), 2001, 133-156. doi:10.1080/13639080020028747

FOOT K. \& GROLEAU C., « Contradictions, transitions, and materiality in organizing processes: An activity theory perspective » in First Monday, 16(6), 2011.

GRAMACCIA G., Les actes de langage dans les organisations. Paris: L'Harmattan, 2001. doi:10.5210/fm.v16i6.3479 
GROLEAU C., «One Phenomenon, Two Lenses: Apprehending Collective Action From the Perspectives of Coorientation and Activity Theories " in Communication as organizing: empirical and theoretical approaches into the dynamic of text and conversation. Mahwah, NJ: Lawrence Erlbaum, 2005.

GROLEAU C., « La syntaxe des artefacts: lier entre elles différentes logiques d'action pour expliquer les pratiques découlant du changement technologique » in Communication E Organisation, (33), 2008, p. 18-28.

HONNETH A., "Visibilité et invisibilité. Sur l'épistémologie de la "reconnaissance” ». in Revue Du MAUSS, 23(1), 2004, 137. doi:10.3917/rdm.023.0137

HONNETH A., "Invisibilité: sur l'épistémologie de la "reconnaissance” » in Réseaux, (1), 2005, 39-57.

HONNETH A., La lutte pour la reconnaissance. Paris Gallimard, 2013.

KAPTELININ V., "The Object of Activity: Making Sense of the Sense-Maker » in Mind, Culture, and Activity, 12(1), 2005, 4-18. doi:10.1207/s15327884mca1201_2

LE BIS I. \& VACHER B., "Les vertus stratégiques de la discrétion des services documentaires: Théories et illustrations " in Documentaliste-Sciences de l'Information, 43(3), 2006. doi:10.3917/docsi.433.0200

LEONTIEV E., Activity, consciousness, and personality. (Marie J. Hall, Trans.). Prentice-Hall, 2009 [1975]. Consulté en ligne, http://www.marxists.org/archive/leontev/ works/1978/index.htm

MIETTINEN R. \& HASU M., «Articulating User Needs in Collaborative Design: Towards an Activity-Theoretical Approach » in Computer Supported Cooperative Work (CSCW), 11(1-2), 129-151, 2002. doi:10.1023/A:1015256909032

NORROS L., "System disturbances as springboard for development of operators' expertise » in Y. Engeström \& D. Middleton (Eds.), Cognition and communication at work (pp. 159-176). Cambridge: Cambridge University Press. 1996.

RUSSELL D. R., « Rethinking Genre in School and Society: An Activity Theory Analysis " in Written Communication, 14(4), 1997, 504-554. doi:10.1177/074108839 7014004004

STRAUSS A., La trame de la négociation - Sociologie qualitative et interactionnisme. Paris: L'Harmattan, 1992.

STRAUSS A. \& CORBIN J., " Grounded Theory Methodology An Overview » in Handbook of qualitative research, 1994.

VYGOTSKY L. S., Mind in society: the development of higher psychological processes. Cambridge: Harvard University Press, 1978 [1934].

WERTSCH J. V., Voices of the mind: a sociocultural approach to mediated action. Cambridge, Mass.: Harvard University Press, 1993.

Résumé : Une recherche dans une organisation de santé implantant un système de Dossier patient numérisé, nous permet d'observer la difficulté à reconnaître le travail d'employés du service d'archivage faisant l'objet de propositions de reclassement. S'ils ont contribué aux anciens processus et participent au projet de rationalisation et 
d'implantation des nouveaux dans le cadre du projet de changement organisationnel, nous observons que la valeur de leur apport s'éteint avec leur participation au projet. La reconnaissance ne peut donc plus se faire en fonction de leur valeur pour le système d'activité, qui redevient théoriquement invisible, intégré dans le système d'information, mais en raison de dimensions morales externes à celui-ci.

Mots-clés : dossier patient, organisation santé, processus organisationnels, projet, reconnaissance, théorie de l'activité, visibilité.

Abstract: Our research in a health organization implementing a Digitized patient records system allows us to observe the difficulty in recognizing the work of employees from the Archives service as they are in the end subject to reclassification proposals. While they have contributed to the former processes and are also involved in the organizational change project and thus helping rationalize and implement the new processes, we observe that the value of their contribution goes out with the end of their participation into the project. Recognition can no longer be based on their value to the activity system, which becomes theoretically invisible as it is integrated into the information system, but falls into external moral dimensions.

Keywords: patient file, health organization, organizational processes, project, recognition, theory of the activity, visibility. 